\title{
Water Fountains in Pre-Planetary Nebulae: The Case of IRAS16342-3814
}

\section{Mark Claussen ${ }^{1}$, Raghvendra Sahai ${ }^{2}$, Mark Morris $^{3}$, and Hannah Rogers ${ }^{1,4}$}

\author{
${ }^{1}$ National Radio Astronomy Observatory, P.O. Box O, 1003 Lopezville Rd., Socorro, NM 87801 \\ ${ }^{2}$ Jet Propulsion Laboratory, 4800 Oak Grove Drive, Pasadena, CA 91109 \\ ${ }^{3}$ University of California, Los Angeles, CA 90095 \\ ${ }^{4}$ Augustana College, 2001 S. Summit Ave, Sioux Falls, SD 57197
}

\begin{abstract}
We present a brief review of Very Long Baseline Array (VLBA) observations of water masers in the so-called water fountain pre-planetary nebulae, and report on new VLBA and Very Large Array (VLA) data for the water masers in the prototypical water fountain source IRAS16342-3814, taken approximately monthly in 2008-2009. A new and very strong water maser is found at an LSR velocity of $-3 \mathrm{~km} \mathrm{~s}^{-1}$, which is offset from the central (likely stellar) velocity. The new VLBA observations still show a similar general structure as was observed in 2002 , but there are details which are difficult to explain.
\end{abstract}

Keywords. Stars: AGB and post-AGB - Masers

\section{Introduction}

Water fountain pre-planetary nebulae $(\mathrm{PPN})$ are a particularly interesting subclass of PPN whose original distinguishing characteristic in the presence of very high-velocity red and blueshifted $\mathrm{H}_{2} \mathrm{O}$ and $\mathrm{OH}$ maser features. Originally the velocity separations of discovered water fountains were in the range of $50-150 \mathrm{~km} \mathrm{~s}^{-1}$, but recently water fountains have been discovered that extend to as much as $500 \mathrm{~km} \mathrm{~s}^{-1}$ (Gómez et al. 2011). When examined with sufficiently high angular resolution (using Very Long Baseline Interferometry - VLBI), or with the VLA, the blue- and red-shifted water masers are typically displaced from each other and show large, opposed, proper motions (e.g. Claussen, Sahai, $\&$ Morris 2009). Lifetimes of the water fountain stage have been estimated to be 50 - 100 years, based on the size and outflow velocity of the maser distribution.

IRAS16342-3814 is a prototypical water fountain source, one of the first three discovered (Likkel \& Morris 1988; Likkel, Morris, \& Maddalena 1992). The discovery water maser spectra are shown in Figure 1. The central LSR velocity is thought to be +43 $\mathrm{kms}^{-1}$, from analysis of water maser "pairs" by Likkel, Morris \& Maddalena 1992 and $\mathrm{OH}$ analysis by Sahai et al. 1999. Observations using the VLBA in 2002 show that the red- and blue-shifted water masers are on opposite sides of the bipolar nebula as seen in scattered light (see Sahai et al. 1999, and are comprised of "bow-shock" features (Figure 2). The groups of extreme velocity masers (both red- and blue-shifted) are situated just outside the optical lobes.

The separation of these extreme velocity features expands with time (see Figure 2). In particular the line joining the most extreme red- and blue-shifted masers keeps its orientation and the length of the line increased over the 5 months of observation (Figure 3 ). A linear least squares fit to the data shown in Figure 3 gives a two-sided expansion proper motion of $63 \pm 2 \mu \mathrm{as} \mathrm{day}^{-1}$ (which translates to 23 mas yr-1). Claussen, Sahai \& Morris 2009 suggested that these detailed structures were actually bow shocks on either side of a 


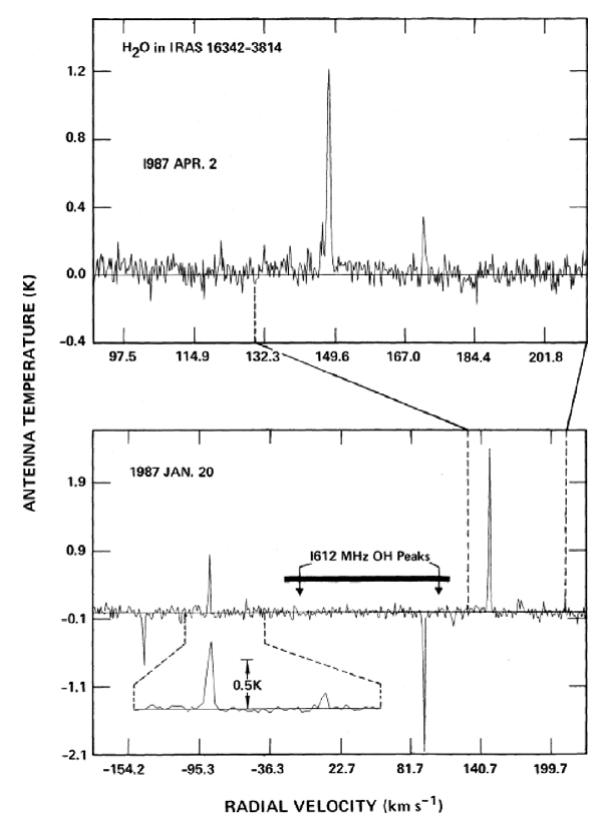

Figure 1. Discovery spectra of water masers from water fountain IRAS16342-3814 (Likkel \& Morris 1988).
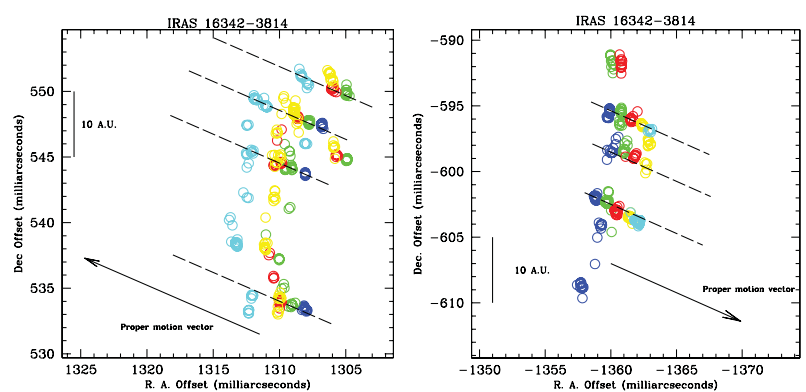

Figure 2. The motion of water masers for 5 monthly epochs in 2002 (the colors denote the different epochs). Left: the motion of water masers for the $+178.7 \mathrm{~km} \mathrm{~s}^{-1}$ radial velocity features. The proper motion vector here represents 14.4 mas $\mathrm{yr}^{-1}$. Right: the motion of water masers for the $-66.0 \mathrm{~km} \mathrm{~s}^{-1}$ radial velocity features. The proper motion vector represents 10.8 mas $\mathrm{yr}^{-1}$.

highly collimated jet. The three-dimensional velocities were found to be $\sim \pm 180 \mathrm{~km} \mathrm{~s}^{-1}$, which leads to a very short dynamical timescale for this source of $\sim 100$ years.

\section{VLBA and VLA Data from 2008-2009}

We observed the water masers from IRAS16342-3814 for twelve epochs beginning in March 2008, spaced approximately monthly. Observations were made with the VLBA and the VLA. For the VLA observations we used seven $6.25 \mathrm{MHz}$ wide frequency settings in the VLA correlator to cover the emission, from LSR velocities -140.0 to $+80.0 \mathrm{~km} \mathrm{~s}^{-1}$ and velocity resolution $0.66 \mathrm{~km} \mathrm{~s}^{-1}$. For the VLBA, we used four $8 \mathrm{MHz}$ baseband channels covering LSR velocities from +230.0 to $-140.0 \mathrm{~km} \mathrm{~s}^{-1}$ and velocity resolution $0.21 \mathrm{~km} \mathrm{~s}^{-1}$. A combined spectrum from the VLA taken on March 26, 2008 is shown in Figure 4. 


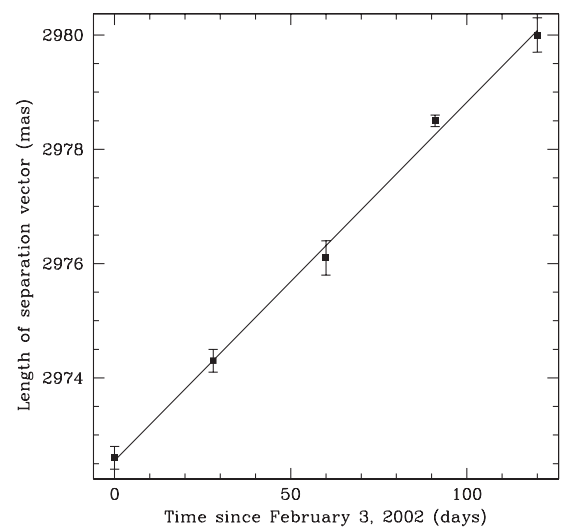

Figure 3. Expansion of line connecting extreme velocity water masers of IRAS16342-3814 in 2002 (Claussen et al. 2009).

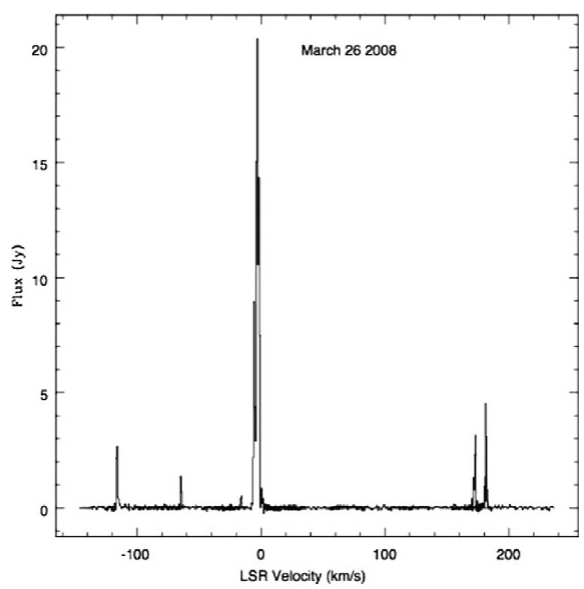

Figure 4. VLA spectrum of water masers of IRAS16342-3814 on March 26, 2008.

\section{Discussion and Future Directions}

In comparing Figure 4 with Figure 1 we see that, in general, the spectra are similar, with red-shifted masers around LSR velocities of $+155 \mathrm{~km} \mathrm{~s}^{-1}$ and $+180 \mathrm{~km} \mathrm{~s}^{-1}$, and blue-shifted masers near an LSR velocity of $-66 \mathrm{~km} \mathrm{~s}^{-1}$. But there are obvious new features as well: the strong masers around $-3 \mathrm{~km} \mathrm{~s}^{-1}$ and a feature at $-118 \mathrm{~km} \mathrm{~s}^{-1}$. The strong feature at $-3 \mathrm{~km} \mathrm{~s}^{-1}$ does not lie at the putative stellar velocity $\left(+43 \mathrm{~km} \mathrm{~s}^{-1}\right.$ with respect to the LSR). Given the new features in this spectrum, however, one might argue that the stellar velocity is closer to $\sim+30 \mathrm{~km} \mathrm{~s}^{-1}$ (centered between the most blue-shifted and the most red-shifted water masers). The new masers at $-3 \mathrm{~km} \mathrm{~s}^{-1}$ are located in approximately the same region as a group of blue-shifted masers $\left(-21\right.$ to $\left.-33 \mathrm{~km} \mathrm{~s}^{-1}\right)$ were found in 2002 (Claussen, Sahai, \& Morris 2009), approximately halfway out from the center to the edge of the southwest optical (scattered light) lobe (Sahai et al. 1999 and Claussen, Sahai, \& Morris 2009).

Although space limits the number of figures allowed for this contribution, the maser structures and distributions for the 2008-2009 observations are similar to those found in 


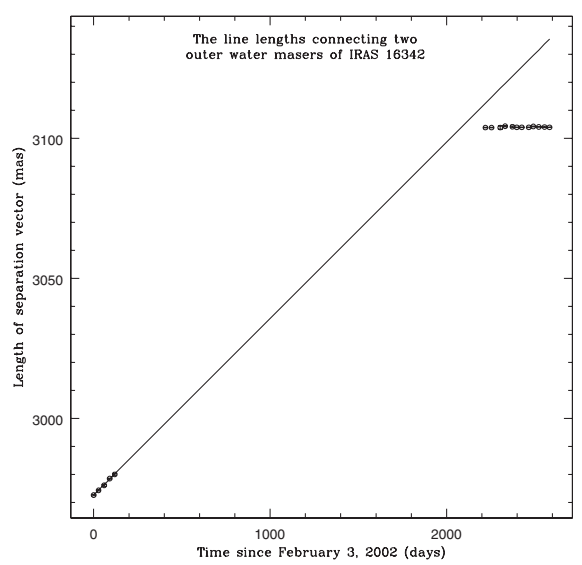

Figure 5. Expansion of line connecting extreme velocity water masers of IRAS16342-3814 in 2002 and 2008-2009.

2002 (Claussen, Sahai \& Morris 2009). The bow-shocked shaped structures seen in the 2002 data, however, are not as clearly defined in the 2008-9 data.

We once again measured the length and position angle of the line joining the +154.2 $\mathrm{km} \mathrm{s}^{-1}$ and $-65.2 \mathrm{~km} \mathrm{~s}^{-1}$ maser features for each epoch in 2008-2009. (We did this because neither project - in 2002 or 2008-2009 used absolute astrometry.) As in 2002, the position angle of this line was unchanging over the 12 months of observation and had the same value as in 2002 , at $66.1^{\circ}$. The results of the line length measurements both in 2002 and 2008-2009 are shown in Figure 5. A very surprising and completely unexpected result was obtained. The length of the line essentially did not change for the 12 epochs in 2008-2009 (the data points in the upper right hand area of Figure 5). The straight line shown in Figure 5 is the extension of the least-squares fit line (of the 2002 data) seen in Figure 3. (In the lower left corner of the figure, the 2002 data can be seen.)

Claussen, Sahai, \& Morris 2009 argue that the proper motion of the water masers in IRAS16342-3814 results from either actual physical motion of the emitting material, or to a "theatre marquee lights" effect, so that dense material at progressively larger distances "light up" sequentially in maser emission as, for example, a passing shock wave produces an outward-moving compression front. These authors argue that for either case, the proper motions of the water masers reflects the physical motion of the dense gas in the jet head, either directly or indirectly. The unexpected result from the 2008-2009 data, that the length of the line joining the (apparently) same two masers is no longer increasing as it was in 2002, may call into question the argument that the masers trace physical motion.

We turn to possible explanations for this unexpected result. We believe that there is no mistake in the reduction of the data; indeed we cannot imagine a problem in data processing that would cause an apparent stoppage of the line length increase. Systematic errors in data processing could cause either a random change in the length of the line (added to the real change in length) or perhaps a large increase in the error itself.

Some other possibilities for the explanation of the change in the (apparent) motion of the water masers are

- The masers really do not reflect physical motion of dense gas, either directly or indirectly. Although this is certainly possible, other observations of masers in different environments have all seemed to be tracing physical motions, and other evidence seems to support this idea (e.g. Bloemhof, Moran, \& Reid 1996). 
- Sometime in the interval between 2002 and 2008, the shock driving the masers reached the edge of the very dense gas in the bipolar nebula. This is a definite possibility, as the positions of the extreme velocity masers, overlaid on the optical nebula, already appeared nearly outside the optical emission. This might explain the effect of an apparent halt to the expansion since the masers seen now are just remnants of earlier shock excitation. In this case one might expect to see some deceleration of the remaining masers. It's not clear that we have seen such deceleration.

Clearly we don't really understand the phenomenon that we have reported in this contribution. Further high angular resolution observations of the water masers in IRAS16342-3814 are likely needed to completely understand this phenomenon that we have seen in 2008-2009. If the second bullet above is a possible explanation, we might expect to see a complete fade-out of the extreme velocity masers.

The National Radio Astronomy Observatory (NRAO) is a facility of the National Science Foundation operated under cooperative agreement by Associated Universities, Inc. Part of this research was carried out under the auspices of the National Science Foundation's Research Experience for Undergraduates (REU) program at the NRAO, and we gratefully acknowledge the funding for this program.

\section{References}

Claussen, M. J., Sahai, R. S., \& Morris, M. R. 2009, ApJ, 691, 219

Gómez, J. F., Rizzo, J. R., Suárez, O., Miranda, L. F., Guerrero, M. A., \& Ramos-Larios, G. 2011, ApJ, 739, 14

Likkel, L. \& Morris, M. 1988, ApJ, 329, 914

Likkel, L., Morris, M. \& Maddalena, R. 1992, A\&AA, 256, 581

Sahai, R., te Lintel Hekkert, P., Morris, M., Zijlstra, A., \& Likkel, L. 1999, ApJ, 514, L115 